\title{
Regularity criteria for 3D shear thinning fluids via two velocity components
}

\section{Jiaqi Yang}

Key Laboratory for Mechanics in Fluid Solid Coupling Systems, Institute of Mechanics, Chinese Academy of Sciences, Beijing 100190, China

\section{A R T I C L E I N F O}

\section{Article history:}

Received 17 October 2018

Received in revised form 15 January 2019

Accepted 19 January 2019

Available online $\mathrm{xxxx}$

\section{Keywords:}

Shear thinning fluids

Regularity criteria

Two velocity components

\section{A B S T R A C T}

Bae \& Choe in a 1997 paper established a regularity criteria for the incompressible Navier-Stokes equations in the whole space $\mathbb{R}^{3}$ based on two velocity components. The goal of this note is to extend their result to the non-Newtonian fluids with shear thinning viscosity.

(c) 2019 Elsevier Ltd. All rights reserved.

\section{Introduction}

In this paper, we are concerned with the non-Newtonian incompressible fluids which is governed by the following system

$$
\begin{cases}\mathbf{u}_{t}+\mathbf{u} \cdot \nabla \mathbf{u}-\operatorname{div} \sigma+\nabla \pi=0, & \text { in } \mathbb{R}^{3} \times(0, T), \\ \operatorname{div} \mathbf{u}=0, & \text { in } \mathbb{R}^{3} \times(0, T), \\ \mathbf{u}(0, x)=\mathbf{u}_{0}(x), & \text { in } \mathbb{R}^{3},\end{cases}
$$

where $\mathbf{u}=\left(u_{1}, u_{2}, u_{3}\right)$ denotes the unknown velocity of the fluids, $\pi$ denotes the pressure, and

$$
\sigma=|D(\mathbf{u})|^{p-2} D(\mathbf{u}), \quad D(\mathbf{u})=\frac{1}{2}\left(\nabla \mathbf{u}+(\nabla \mathbf{u})^{T}\right)
$$

denotes the shear stress. Since we are concerned with the study of sufficient condition for regularity of weak solutions to the above system, we start by giving the well known definitions of weak and strong solutions to (1).

Definition 1 ([1,2]). Let $\mathbf{u}_{0} \in L_{\sigma}^{2}\left(\mathbb{R}^{3}\right)$. We call $\mathbf{u} \in L^{\infty}\left(0, T ; L_{\sigma}^{2}\left(\mathbb{R}^{3}\right)\right) \cap L^{p}\left(0, T ; W_{0, \sigma}^{1, p}\left(\mathbb{R}^{3}\right)\right)$ a weak solution of $(1)$, if

$$
\begin{aligned}
& \int_{\mathbb{R}^{3} \times(0, T)}-\mathbf{u} \cdot \boldsymbol{\varphi}_{t} d x d t-\int_{\mathbb{R}^{3} \times(0, T)} \mathbf{u} \otimes \mathbf{u}: \nabla \boldsymbol{\varphi} d x d t \\
& \quad+\int_{\mathbb{R}^{3} \times(0, T)}|D(\mathbf{u})|^{p-2} D(\mathbf{u}): D(\varphi) d x d t=\int_{\mathbb{R}^{3}} \mathbf{u}_{0} \cdot \boldsymbol{\varphi}(0) d x
\end{aligned}
$$

for all $\varphi \in C_{0}^{\infty}\left(\mathbb{R}^{3} \times[0, T)\right)$ satisfying $\operatorname{div} \varphi=0$ and, furthermore, if the following energy inequality

$$
\frac{1}{2}\|\mathbf{u}(t)\|_{2}^{2}+\int_{0}^{t} \int_{\mathbb{R}^{3}}|D(\mathbf{u})|^{p} d x d t \leq \frac{1}{2}\left\|\mathbf{u}_{0}\right\|_{2}^{2},
$$

holds for almost all $t \in(0, T)$.

E-mail address: yjq@imech.ac.cn. 
We use the following notations and definitions: $C_{0}^{\infty}\left(\mathbb{R}^{3} \times[0, T)\right)$ and $C_{0}^{\infty}\left(\mathbb{R}^{3}\right)$ denote two spaces of smooth functions with compact support, and $C_{0, \sigma}^{\infty}\left(\mathbb{R}^{3}\right)=\left\{\boldsymbol{\varphi} \in C_{0}^{\infty}\left(\mathbb{R}^{3}\right) \mid \nabla \cdot \boldsymbol{\varphi}=0\right\}$. The norm in $L^{p}\left(\mathbb{R}^{3}\right)$ will be denoted by $\|\cdot\|_{p}$, and

$$
\|\cdot\|_{W^{k, p}}:=\sum_{0 \leq|\alpha| \leq k}\left\|\partial^{\alpha}(\cdot)\right\|_{p}, \quad k \geq 1 .
$$

$L_{\sigma}^{p}\left(\mathbb{R}^{3}\right)$ denotes the closure of $C_{0, \sigma}^{\infty}\left(\mathbb{R}^{3}\right)$ in the norm $\|\cdot\|_{p}$, and $W_{0, \sigma}^{1, p}\left(\mathbb{R}^{3}\right)$ the closure of $C_{0, \sigma}^{\infty}\left(\mathbb{R}^{3}\right)$ in the norm $\|\cdot\|_{W^{1, p}}$.

Definition 2 ([3]). Let $\mathbf{u}_{0} \in W_{0, \sigma}^{1,2}\left(\mathbb{R}^{3}\right)$. We say that a weak solution $\mathbf{u}$ to (1) is strong if

$$
\mathbf{u} \in L^{p}\left(0, T ; W^{2, p}\left(\mathbb{R}^{3}\right)\right) \cap L^{\infty}\left(0, T ; W_{0, \sigma}^{1,2}\left(\mathbb{R}^{3}\right)\right) .
$$

The bibliography concerning the system (1) is too large to be dealt here. So, the aim of our references is merely to help the reading of the notes. In particular, we essentially restrict us to the evolution problem, in the case $p \leq 2$.

For the mathematical study of Eq. (1) one must recall the pioneering study done by O.A. Ladyzhenskaya in reference [4] where, in particular, existence of strong global solutions with periodic boundary conditions is proved for $p \geq \frac{11}{5}$. The existence of weak solutions of (1) is shown in [4] with the periodic boundary condition, and in [5] in the whole space for $p>\frac{9}{5}$. In [2], J. Wolf showed the existence of weak solutions with Dirichlet boundary condition for $p>\frac{8}{5}$. For $\frac{7}{5}<p \leq 2$, i.e. in the shear thinning case, the short time existence results of strong solutions with the periodic boundary condition or in the whole space, are established in [6,7]. Moreover, when $\frac{8}{5}<p \leq 2$, Bae et al. [3] obtained the following Prodi-Serrin type regularity criteria.

Lemma 1 (Theorem 2.3, [3]). Let $\frac{8}{5}<p \leq 2$. Suppose that $\mathbf{u}$ is a weak solution to (1) in $[0, T)$. Assume further that

$$
\mathbf{u} \in L^{\beta}\left(0, T ; L^{\alpha}\left(\mathbb{R}^{3}\right)\right), \quad \frac{3}{\alpha}+\frac{5 p-6}{2 \beta} \leq \frac{5 p-8}{2}, \quad \alpha>\frac{6}{5 p-8} .
$$

Then $\mathbf{u}$ is a strong solution to (1) in $[0, T)$.

For a related shear thickening fluids result, $2<p<\frac{11}{5}$, see reference [1].

When $p=2$, the system (1) reduces to the classical Navier-Stokes equations. In this case the global existence of weak solutions goes back to Leray [8] and Hopf [9]. It is one of the most challenging open mathematical problems to prove, or disprove, that weak solutions are necessarily strong under reasonable but general assumptions. In this context, a remarkable and classical sufficient condition for uniqueness and regularity is the so-called Prodi-Serrin condition, namely

$$
\mathbf{u} \in L^{\beta}\left(0, T ; L^{\alpha}\left(\mathbb{R}^{3}\right)\right), \quad \frac{2}{\beta}+\frac{3}{\alpha} \leq 1, \quad \alpha>3 .
$$

Weak solutions satisfying the condition (3) are known to be strong and unique, see [10-13]. In [14], see also [15], the authors proved, in the whole space case, that a solution is strong if merely two components of the velocity satisfy the above condition. Very recently, this result was extended to the half-space in [16], and cylindrical boundaries in [17], both cases under slip boundary conditions. Below we extend the result proved in [3] to the system (1) with $p<2$, i.e., we will improve Lemma 1. Our main result reads as follows.

Theorem 1. Let $\mathbf{u}$ be a weak solution to the system (1) with $\frac{8}{5}<p \leq 2$. Furthermore, let $\overline{\mathbf{u}}=\left(u_{1}, u_{2}, 0\right)$, and assume that

$$
\overline{\mathbf{u}} \in L^{\beta}\left(0, T ; L^{\alpha}\left(\mathbb{R}^{3}\right)\right), \quad \frac{3}{\alpha}+\frac{5 p-6}{2 \beta} \leq \frac{5 p-8}{2}, \quad \alpha>\frac{6}{5 p-8} .
$$

then $\mathbf{u}$ is a strong solution.

Remark 1. Following [3], and from the following proof, we may show that the above result still holds when $\overline{\mathbf{u}} \in$ $L^{\infty}\left(0, T ; L^{\frac{6}{5 p-8}}\left(\mathbb{R}^{3}\right)\right)$ and $\|\overline{\mathbf{u}}\|_{L^{\infty}\left(0, T ; L^{5 p-8}\right)}$ is sufficiently small.

Remark 2. Note that if $p=2$ we reobtain the result shown in reference [14].

Remark 3. The above choice $\bar{u}=\left(u_{1}, u_{2}, 0\right)$ is equivalent to say that $\bar{u}$ is a (two dimensional) component of the velocity parallel to a fixed, arbitrary plane.

\section{Preliminary results}

Lemma 2 ([18,19], Korn inequality). Let $\mathbf{u} \in W^{1, p}\left(\mathbb{R}^{3}\right)$, for $1<p<\infty$. Then

$$
\|\nabla \mathbf{u}\|_{p} \leq C\|D(\mathbf{u})\|_{p} .
$$

Hereinafter, we define $C$ as being a generic constant which value may vary line by line. 
Lemma 3 ([18,20], generalized Korn inequality). Let $\mathbf{u} \in W^{2, p}\left(\mathbb{R}^{3}\right)$, for $1<p<\infty$. Then

$$
\left\|\nabla^{2} \mathbf{u}\right\|_{p} \leq C\|\nabla D(\mathbf{u})\|_{p} .
$$

By virtue of Lemmas 2 and 3, and noting that

$$
\sum_{i, j=1}^{3} \nabla\left(|D|^{p-2} D_{i j}\right) \cdot \nabla D_{i j} \geq(p-1) \int|D|^{p-2}|\nabla D|^{2} d x,
$$

a straightforward calculation implies the following lemma, the details can be found in Lemma 2.1 of [3].

Lemma 4. Let $1<p<2$. Suppose $\mathbf{u} \in W^{2, s}\left(\mathbb{R}^{3}\right)$ for $1<s<2$, then

$$
\begin{aligned}
\int_{\mathbb{R}^{3}}\left|\nabla^{2} \mathbf{u}\right|^{s} d x & \leq C\left(\int_{\mathbb{R}^{3}} \sum_{i, j=1}^{3} \nabla\left(|D|^{p-2} D_{i j}\right) \cdot \nabla D_{i j} d x\right)^{\frac{s}{2}}\left(\int_{\mathbb{R}^{3}}|\nabla \mathbf{u}|^{\frac{(2-p) s}{2-s}} d x\right)^{\frac{2-s}{2}} \\
& \leq C \int_{\mathbb{R}^{3}} \sum_{i, j=1}^{3} \nabla\left(|D|^{p-2} D_{i j}\right) \cdot \nabla D_{i j} d x+C \int_{\mathbb{R}^{3}}|\nabla \mathbf{u}|^{\frac{(2-p) s}{2-s}} d x .
\end{aligned}
$$

Hereinafter, for convenience, we set $D=D(\mathbf{u})$ and $D_{i j}=\frac{\partial_{i} u_{j}+\partial_{j} u_{i}}{2}$.

\section{Proof of Theorem 1}

Proof. Applying the inner product $\nabla \times(\nabla \times \mathbf{u})$ to (1), we have

$$
\int_{\mathbb{R}^{3}}\left(\mathbf{u}_{t}+\mathbf{u} \cdot \nabla \mathbf{u}-\operatorname{div}\left(|D|^{p-2} D\right)\right) \cdot(\nabla \times(\nabla \times \mathbf{u})) d x=0 .
$$

After integrating by parts, we easily obtain

$$
\frac{1}{2} \frac{d}{d t} \int_{\mathbb{R}^{3}}|\omega|^{2} d x+\sum_{i, j=1}^{3} \int_{\mathbb{R}^{3}} \nabla\left(|D|^{p-2} D_{i j}\right) \cdot \nabla D_{i j} d x=\int_{\mathbb{R}^{3}} \boldsymbol{\omega} \cdot \nabla \mathbf{u} \cdot \boldsymbol{\omega} d x
$$

where $\omega=\nabla \times \mathbf{u}$. We have used the following facts:

$$
-\Delta \mathbf{u}=\nabla \times(\nabla \times \mathbf{u}), \quad \mathbf{u} \cdot \nabla \mathbf{u}=\frac{1}{2} \nabla|\mathbf{u}|^{2}-\mathbf{u} \times(\nabla \times \mathbf{u}),
$$

and

$$
\nabla \times(\mathbf{u} \times \boldsymbol{\omega})=\boldsymbol{\omega} \cdot \nabla \mathbf{u}-\mathbf{u} \cdot \nabla \boldsymbol{\omega} .
$$

Hence, we have

$$
\frac{1}{2} \frac{d}{d t} \int_{\mathbb{R}^{3}}|\omega|^{2} d x+\sum_{i, j=1}^{3} \int_{\mathbb{R}^{3}} \nabla\left(|D|^{p-2} D_{i j}\right) \cdot \nabla D_{i j} d x \leq\left|\sum_{i, j=1}^{3} \int_{\mathbb{R}^{3}} \omega_{j} \partial_{j} u_{i} \omega_{i} d x\right| .
$$

Following [14] or [16], we consider separately the three cases $i \neq 3 ; i=3$ and $j \neq 3 ; i=j=3$.

Case $\mathrm{I}: i \neq 3$. By integration by parts, one has

$$
\sum_{j=1}^{3} \int_{\mathbb{R}^{3}} \omega_{j}\left(\partial_{j} u_{i}\right) \omega_{i} d x=-\sum_{j=1}^{3} \int_{\mathbb{R}^{3}} u_{i} \omega_{j} \partial_{j} \omega_{i} d x
$$

Case II: $i=3$ and $j \neq 3$. By integration by parts, one has

$$
\begin{aligned}
\sum_{j=1}^{2} \int_{\mathbb{R}^{3}} \omega_{j}\left(\partial_{j} u_{3}\right) \omega_{3} d x= & \sum_{j=1}^{2} \int_{\mathbb{R}^{3}} \omega_{j} \partial_{j} u_{3}\left(\partial_{1} u_{2}-\partial_{2} u_{1}\right) d x \\
= & -\sum_{j=1}^{2} \int_{\mathbb{R}^{3}} u_{2}\left(\partial_{1} \omega_{j} \partial_{j} u_{3}+\omega_{j} \partial_{j} \partial_{1} u_{3}\right) d x \\
& +\sum_{j=1}^{2} \int_{\mathbb{R}^{3}} u_{1}\left(\partial_{2} \omega_{j} \partial_{j} u_{3}+\omega_{j} \partial_{j} \partial_{2} u_{3}\right) d x
\end{aligned}
$$


Case III: $i=j=3$. Due to $\operatorname{div} \mathbf{u}=0$, one has

$$
\begin{aligned}
\int_{\mathbb{R}^{3}}\left(\partial_{3} u_{3}\right) \omega_{3}^{2} d x & =-\int_{\mathbb{R}^{3}}\left(\partial_{1} u_{1}+\partial_{2} u_{2}\right) \omega_{3}^{2} d x \\
& =2 \int_{\mathbb{R}^{3}} u_{1} \omega_{3} \partial_{1} \omega_{3} d x+2 \int_{\mathbb{R}^{3}} u_{2} \omega_{3} \partial_{2} \omega_{3} d x .
\end{aligned}
$$

Now, from (5)-(7), we have

$$
\begin{aligned}
& \frac{1}{2} \frac{d}{d t} \int_{\mathbb{R}^{3}}|\omega|^{2} d x+\sum_{i, j=1}^{3} \int_{\mathbb{R}^{3}} \nabla\left(|D|^{p-2} D_{i j}\right) \cdot \nabla D_{i j} d x \\
& \leq C \int_{\mathbb{R}^{3}}\left|\overline{\mathbf{u}}\|\nabla \mathbf{u}\| \nabla^{2} \mathbf{u}\right| d x .
\end{aligned}
$$

On the other hand, since $\alpha>\frac{6}{5 p-8}>\frac{3 p}{4 p-6}>\frac{p}{p-1}$, we have

$$
\begin{aligned}
& \int_{\mathbb{R}^{3}}\left|\overline{\mathbf{u}}\|\nabla \mathbf{u}\| \nabla^{2} \mathbf{u}\right| d x \leq\|\overline{\mathbf{u}}\|_{\alpha}\left\||\nabla \mathbf{u}|\left|\nabla^{2} \mathbf{u}\right|\right\|_{\frac{\alpha}{\alpha-1}} \\
& \leq\|\overline{\mathbf{u}}\|_{\alpha}\|\nabla \mathbf{u}\|_{\frac{p \alpha}{p \alpha-p-\alpha}}\left\|\nabla^{2} \mathbf{u}\right\|_{p} \\
& \leq\|\overline{\mathbf{u}}\|_{\alpha}\|\nabla \mathbf{u}\|_{2}^{\frac{8 p \alpha-12 \alpha-6 p}{(5 p-6) \alpha}}\|\nabla \mathbf{u}\| \frac{\frac{3 p}{3-p}}{\frac{3(2 p+2 \alpha-p \alpha) \alpha}{(5 p-p)}}\left\|\nabla^{2} \mathbf{u}\right\|_{p} \\
& \leq C\|\overline{\mathbf{u}}\|_{\alpha}\|\nabla \mathbf{u}\|_{2}^{\frac{8 p \alpha-12 \alpha-6 p}{(5 p-6) \alpha}}\left\|\nabla^{2} \mathbf{u}\right\|_{p}^{\frac{2 p p \alpha+6 p}{(5 p-6) \alpha}} \text {. }
\end{aligned}
$$

Hence, for any $0 \leq t_{1}<t_{2} \leq T$, we have

$$
\begin{aligned}
& \int_{t_{1}}^{t_{2}} \int_{\mathbb{R}^{3}}\left|\overline{\mathbf{u}}\|\nabla \mathbf{u}\| \nabla^{2} \mathbf{u}\right| d x d t \\
& \quad \leq C\left(\int_{t_{1}}^{t_{2}}\|\overline{\mathbf{u}}\|_{\alpha}^{\beta} d t\right)^{\frac{1}{\beta}}\left(\int_{t_{1}}^{t_{2}}\|\nabla \mathbf{u}\|_{2}^{\frac{\beta}{\beta-1} \frac{8 p \alpha-12 \alpha-6 p}{(5 p-6) \alpha}}\left\|\nabla^{2} \mathbf{u}\right\|_{p}^{\frac{\beta}{\beta-1} \frac{2 p \alpha+6 p}{(5 p-6) \alpha}} d t\right)^{\frac{\beta-1}{\beta}} \\
& \quad \leq C\left(\int_{0}^{T}\|\overline{\mathbf{u}}\|_{\alpha}^{\beta} d t\right)^{\frac{1}{\beta}} \sup _{t \in\left[t_{1}, t_{2}\right]}\|\nabla \mathbf{u}\|_{2}^{\frac{8 p \alpha-12 \alpha-6 p}{(5 p-6) \alpha}}\left(\int_{t_{1}}^{t_{2}}\left\|\nabla^{2} \mathbf{u}\right\|_{p}^{\frac{\beta}{\beta-1}(5 p-6) \alpha} d t\right)^{\frac{2 p-1}{\beta}} .
\end{aligned}
$$

Due to (4), we have

$$
\frac{\beta}{\beta-1} \frac{2 \alpha+6}{(5 p-6) \alpha} \leq 1 \text {. }
$$

Therefore

$$
\begin{aligned}
& \int_{t_{1}}^{t_{2}} \int_{\mathbb{R}^{3}}\left|\overline{\mathbf{u}}\|\nabla \mathbf{u}\| \nabla^{2} \mathbf{u}\right| d x d t \\
\leq & C\left(\int_{t_{1}}^{t_{2}}\|\overline{\mathbf{u}}\|_{\alpha}^{\beta} d t\right)^{\frac{1}{\beta}} \sup _{t \in\left[t_{1}, t_{2}\right]}\|\nabla \mathbf{u}\|_{2}^{\frac{8 p \alpha-12 \alpha-6 p}{(5 p-6) \alpha}}\left(\int_{t_{1}}^{t_{2}}\left\|\nabla^{2} \mathbf{u}\right\|_{p}^{p} d t\right)^{\frac{2 \alpha+6}{(5 p-6) \alpha}} .
\end{aligned}
$$

In Lemma 4 , set $s=p$. By appealing to (2) and Lemma 2, we have

$$
\begin{aligned}
& \int_{t_{1}}^{t_{2}} \int_{\mathbb{R}^{3}}\left|\overline{\mathbf{u}}\|\nabla \mathbf{u}\| \nabla^{2} \mathbf{u}\right| d x d t \\
\leq & C\left(\int_{t_{1}}^{t_{2}}\|\overline{\mathbf{u}}\|_{\alpha}^{\beta} d t\right)^{\frac{1}{\beta}} \sup _{t \in\left[t_{1}, t_{2}\right]}\|\nabla \mathbf{u}\|_{2}^{\frac{8 p \alpha-12 \alpha-6 p}{(5 p-6) \alpha}}\left(\int_{t_{1}}^{t_{2}}\|\nabla \mathbf{u}\|_{p}^{p} d t\right)^{\frac{(2-p)(\alpha+3)}{5 p-6}} \\
& \times\left(\int_{t_{1}}^{t_{2}} \int_{\mathbb{R}^{3}} \sum_{i, j=1}^{3} \nabla\left(|D|^{p-2} D_{i j}\right) \cdot \nabla D_{i j} d x d t\right)^{\frac{p(\alpha+3)}{5 p-6}} \\
\leq & C\left(\int_{t_{1}}^{t_{2}}\|\overline{\mathbf{u}}\|_{\alpha}^{\beta} d t\right)^{\frac{1}{\beta}} \sup _{t \in\left[t_{1}, t_{2}\right]}\|\nabla \mathbf{u}\|_{2}^{\frac{8 p \alpha-12 \alpha-6 p}{(5 p-6) \alpha}} \\
& \times\left(\int_{t_{1}}^{t_{2}} \int_{\mathbb{R}^{3}} \sum_{i, j=1}^{3} \nabla\left(|D|^{p-2} D_{i j}\right) \cdot \nabla D_{i j} d x d t\right)^{\frac{p(\alpha+3)}{(5 p-6) \alpha}} .
\end{aligned}
$$


Thus, from (8) and (9), it follows that

$$
\begin{aligned}
& \left\|\nabla \mathbf{u}\left(t_{2}\right)\right\|_{2}^{2}+\int_{t_{1}}^{t_{2}} \int_{\mathbb{R}^{3}} \sum_{i, j=1}^{3} \nabla\left(|D|^{p-2} D_{i j}\right) \cdot \nabla D_{i j} d x d t \\
\leq & C\left(\int_{t_{1}}^{t_{2}}\|\overline{\mathbf{u}}\|_{\alpha}^{\beta} d t\right)^{\frac{1}{\beta}} \sup _{t \in\left[t_{1}, t_{2}\right]}\|\nabla \mathbf{u}\|_{2}^{\frac{8 p \alpha-12 \alpha-6 p}{(5 p-6) \alpha}} \\
& \times\left(\int_{t_{1}}^{t_{2}} \int_{\mathbb{R}^{3}} \sum_{i, j=1}^{3} \nabla\left(|D|^{p-2} D_{i j}\right) \cdot \nabla D_{i j} d x d t\right)^{\frac{p(\alpha+3)}{(5 p-6) \alpha}}+\left\|\nabla \mathbf{u}\left(t_{1}\right)\right\|_{2}^{2} .
\end{aligned}
$$

Since $\overline{\mathbf{u}} \in L^{\beta}\left(0, T ; L^{\alpha}\left(\mathbb{R}^{3}\right)\right)$ and $\frac{4 p \alpha-6 \alpha-3 p}{(5 p-6) \alpha}+\frac{p(\alpha+3)}{(5 p-6) \alpha}=1$, we have

$$
\sup _{t \in[0, T]}\|\nabla \mathbf{u}(t)\|_{2}^{2}+\int_{0}^{T} \int_{\mathbb{R}^{3}} \sum_{i, j=1}^{3} \nabla\left(|D|^{p-2} D_{i j}\right) \cdot \nabla D_{i j} d x d t \leq\left\|\nabla \mathbf{u}_{0}\right\|_{2}^{2} .
$$

See the proof of Theorem 2.3 in [3] for the details. Now from Lemma 4, one has

$$
\sup _{t \in[0, T]}\|\nabla \mathbf{u}(t)\|_{2}^{2}+\int_{0}^{T} \int_{\mathbb{R}^{3}}\left|\nabla^{2} \mathbf{u}\right|^{p} d x d t \leq C \int_{0}^{T} \int_{\mathbb{R}^{3}}|\nabla \mathbf{u}|^{p} d x d t+\left\|\nabla \mathbf{u}_{0}\right\|_{2}^{2} \leq C .
$$

Thus, the proof of Theorem 1 is complete.

\section{Acknowledgment}

I thank the anonymous referee for giving me many helpful suggestions and comments which led to improvement of the presentation.

\section{References}

[1] H.-O. Bae, K. Kang, J. Lee, J. Wolf, Regularity for Ostwald-de Waele type shear thickening fluids, NoDEA Nonlinear Differential Equations Appl. 22 (2015) 1-19.

[2] J. Wolf, Existence of weak solutions to the equations of non-stationary motion of non-Newtonian fluids with shear rate dependent viscosity, J. Math. Fluid Mech. 9 (2007) 104-138.

[3] H.-O. Bae, H.J. Choe, D.W. Kim, Regularity and singularity of weak solutions to Ostwald-de Waele flows, in: International Conference on Differential Equations and Related Topics (Pusan, 1999), Vol. 37, (2000) J. Korean Math. Soc., 1999, pp. 957-975.

[4] O.A. Ladyzhenskaya, The Mathematical Theory of Viscous Incompressible Flow, second ed., Gordon and Breach, New York, 1969.

[5] M. Pokorny, Cauchy problem for the non-Newtonian viscous incompressible fluid, Appl. Math. 41 (1996) 169-201.

[6] L.C. Berselli, L. Diening, M. Ruzicka, Existence of strong solutions for incompressible fluids with shear dependent viscosities, J. Math. Fluid Mech. 12 (2010) 101-132.

[7] L. Diening, M. Ruzicka, Strong solutions for generalized Newtonian fluids, J. Math. Fluid Mech. 7 (2005) 413-450.

[8] J. Leray, Sur le mouvement d'un liquide visqueux emplissant l'espace, Acta Math. 63 (1934) 193-248.

[9] E. Hopf, über die anfangswertaufgabe für die hydrodynamischen Grundgleichungen, Math. Nachr. 4 (1951) 213-231.

[10] C. Foias, Une remarque sur l'unicité des solutions des équations de Navier-Stokes en dimension n, Bull. Soc. Math. Fr. 89 (1961) 1-8.

[11] G. Prodi, Un teorema di unicità per le equazioni di Navier-Stokes, Ann. Mat. Pura Appl. 48 (1959) 173-182, Italian.

[12] J. Serrin, On the interior regularity of weak solutions of the Navier-Stokes equations, Arch. Ration. Mech. Anal. 9 (1962) $187-195$.

[13] J. Serrin, The initial value problem for the Navier-Stokes equations, in: R.E. Langer (Ed.), Nonlinear Problems, University of Wisconsin Press, Madison, 1963, pp. 69-98.

[14] H.-O. Bae, H.J. Choe, $L^{\infty}$-bound of weak solutions to Navier-Stokes equations, in: Proceedings of the Korea-Japan Partial Differential Equations Conference (Taejon, 1996), in: Lecture Notes Ser., vol. 39, Seoul Nat. Univ. Seoul, 1997, p. 13.

[15] H.-O. Bae, H.J. Choe, A regularity criterion for the Navier-Stokes equations, Comm. Partial Differential Equations 32 (2007) $1173-1187$.

[16] H. Beirão da Veiga, On the extension to slip boundary conditions of a Bae and Choe regularity criterion for the Navier-Stokes equations. The half space case, J. Math. Anal. Appl. 453 (2017) 212-220.

[17] H. Beirão da Veiga, J. Bemelmans, J. Brand, On a two components condition for regularity of the 3D Navier-Stokes equations under physical slip boundary conditions on non-flat boundaries, Math. Ann. (2018) 1-38.

[18] H. Bellout, F. Bloom, J. Nečas, Young measure-valued solutions for non-newtonian incompressible fluids, Comm. Partial Differential Equations 19 (1994) 1763-1803.

[19] V.A. Kondrat'ev, O.A. Olenik, Boundary value problems for a system in elasticity theory in unbounded domains, Korn inequalities, Russ. Math. Surv. 43 (1988) 65-119.

[20] H. Bellout, F. Bloom, J. Nečas, Existence, uniqueness, And stability of solutions to the initial-boundary value problem for bipolar viscous fluids, Differential Integral Equations 8 (1995) 453-464. 